\section{Control in chronic infections}

The identities and actions of molecular and cellular mediators of the immune response during chronic viral infections remain poorly understood. In Science, Zuniga and colleagues report that interleukin 6 (IL-6) is essential for viral control during chronic infection with clone 13 lymphocytic choriomeningitis virus (LCMV). IL-6 produced by follicular dendritic cells late in infection is required for the increase in $T$ follicular helper $\left(T_{\mathrm{FH}}\right)$ cell numbers observed in chronic clone 13 infections and consequently for germinal-center formation and LCMV-specific antibody production. Mechanistically, IL- 6 signals in $\mathrm{CD}^{+}{ }^{+} \mathrm{T}$ cells in a cell-intrinsic manner to upregulate $\mathrm{Bcl}-6$, a transcription factor required for $T_{F H}$ differentiation. Despite the loss of $T_{F H}$ cells in IL-6-deficient mice, IL-21 remains unchanged, suggesting that there are redundant sources for this cytokine in vivo. IL-6deficient mice efficiently clear acute (Armstrong clone) LCMV infections.

Science (29 September 2011) doi:10.1126/science.1208421

\section{Mast cells crack down on venom}

Many animal venoms contain factors that are able to trigger mast cell degranulation. In the Journal of Clinical Investigation, Galli and colleagues demonstrate that degranulation is critical for mitigating envenomation. The venom of the lizard Heloderma suspectum (the Gila monster) contains helodermin, a venom structurally similar to mammalian vasoactive intestinal peptide (VIP) — an important trigger of mast cell degranulation. Helodermin also triggers mast cell degranulation and is responsible for the symptoms of envenomation. Using mast celldeficient mice and by mast cell grafting, the authors show that these cells are critical for protection against helodermin through their specific action at the site of venom challenge. Protection is mediated by the mast cell factor MCPT4, which degrades both helodermin and VIP, suggesting that MCPT4 is important in controlling not only venom toxicity but also physiological regulation of endogenous VIP.

J. Clin. Invest. 121, 4180-4191 (2011)

\section{Vegetable boost to IELs}

Intestinal epithelial lymphocytes (IELs) are responsible for maintaining gut barrier function as well as regulating the microbial burden in the lumen. In Cell Veldhoen and colleagues reveal that diet-derived compounds can in turn regulate IEL populations, with important consequences for immunohomeostasis. They observe that IELs have high basal amounts of the xenobiotic sensor aryl hydrocarbon receptor (AhR). The absence of AhR does not have any generalized effects on IELs but rather results in their specific loss from mucosal surfaces. The authors identify the phytochemical I3C as an important dietary source of AhR ligands. Dietary exclusion of I3C reduces mucosal populations of IELs and leads to perturbed microbial colonization and exacerbated gut inflammation. I3C and related compounds are particularly abundant in cruciferous vegetables such as cabbages, and these findings therefore reveal a direct mechanistic link between diet, intestinal immunity and the microbiota. Cell (11 October 2011) doi:10.1016/j.cell.2011.09.025

Written by Laurie A. Dempsey, Zoltan Fehervari \& Ioana Visan

\section{Malaria insights}

The mechanisms involved in the development of cerebral malaria remain poorly defined. In the Journal of Experimental Medicine, Mecheri and colleagues show that a subset of $\mathrm{FcERI}^{+}$neutrophils home to the brain and are responsible for mediating the development of cerebral malaria in mice infected with Plasmodium berghei ANKA. Infected mice have an increase in the proportion of FceRI ${ }^{+}$neutrophils in the brain 6-8 days after infection, shortly before their death due to cerebral complications. Naive neutrophils are FceRI negative, but $P$. berghei ANKA infection induces the expression of FceRI $\alpha$ and $\gamma$ subunits in these cells. FceRI $\alpha$-deficient, IgE-deficient or neutrophil-depleted mice are resistant to experimentally induced cerebral malaria. Interferon- $\gamma$ and IL- 6 are decreased in infected FceRI $\alpha$-deficient mice, indicating that FceRI ${ }^{+}$neutrophils modulate the inflammatory response in the brain.

J. Exp. Med (3 October 2011) doi:10.1084/jem.20110845

\section{Reorienting the groove}

It is known that CD1 molecules can present a variety of glycolipids to natural killer T cells, but how differences in the lipid chains influence TCR recognition and why non-polymorphic CD1 molecules can accommodate extensive lipid diversity remain puzzling. In the Proceedings of the National Academy of Science, Garcia-Alles et al. provide hints by presenting the structure of human CD1b complexed with a sulfoglycolipid from Mycobacterium tuberculosis. They find that endogenous alkyldiacylglycerol lipids occupy lipid-binding grooves in the CD1b molecule, acting as 'spacers' or placeholders for exogenous lipids. Upon antigen loading, these spacers shift within the CD1b and lead to reorientation of $A^{\prime}$ and $F^{\prime}$ lipid-binding pockets. CD1b residues $149-152$, located at the $F^{\prime}$ pocket-TCR interface, shift by several angstroms and govern access to this pocket. These changes allow CD1b to bind bulky polar headgroups but limit the length of the alkyl chains that can be accommodated by the $A^{\prime}$ pocket. This structural plasticity explains the ability of CD1b to present multiple glycolipids.

Proc. Natl. Acad. Sci. USA (17 October 2011)

doi:10.1073/pnas. 1110118108

\section{Caspase-11 inflammasomes}

Various NOD-like receptors can sense intracellular pathogens or danger and assemble inflammasomes that activate the protease caspase- 1 and leads to mature interleukin $1 \beta$ (IL-1 $\beta$ ) and IL-18 secretion. In Nature, Dixit and colleagues identify a role for mouse caspase-11 in response to cholera toxin B and infection by enteric bacteria. Casp11 is closely linked to Casp 1 in the genome. Mouse strain 129 and 129-derived embryonic stem cells lack expression of caspase-11 due to a 5-base-pair deletion that disrupts exon splicing. Targeted $\mathrm{Casp1}^{-/-}$mice actually lack expression of both caspases, prompting a reassessment of their roles in IL-1 $\beta$ and IL-18 activation as well as induction of apoptosis. Although caspase-1 activation by ATP and uric acid remains intact, caspase- 11 activation is mediated by a noncanonical inflammasome that does not require NLRP3 or ASC. Mice lacking caspase-11, but not caspase-1, alone survive lethal lipopolysaccharide challenge. It remains unknown what targets of caspase-11 mediate the tissue damage seen in septic shock; however, these findings suggest that caspase- 1 is not the relevant executor. These results complicate interpretation of previous results implicating caspase- 1 activity in sepsis.

$L A D$

Nature (16 October 2011) doi:10.1038/nature10558 